Acta Crystallographica Section C

Crystal Structure

Communications

ISSN 0108-2701

\title{
3-Anilino- $\mathrm{N}$-phenyl-1 $\mathrm{H}$-indole-2-carbothioamide
}

L. Jing, F. He-Liang, S. Jie and C. Wei-Xing

This electronic document was scanned from an archival copy of material deposited to accompany a paper published in an IUCr journal. In many cases the only accessible copy was a microfilm of a poor-quality original. 
CH10e reused

10|F|O vS 10|F|C

$k$. FO FC sigF $k$ I FO FC sigFlk 1 FO FC sigF

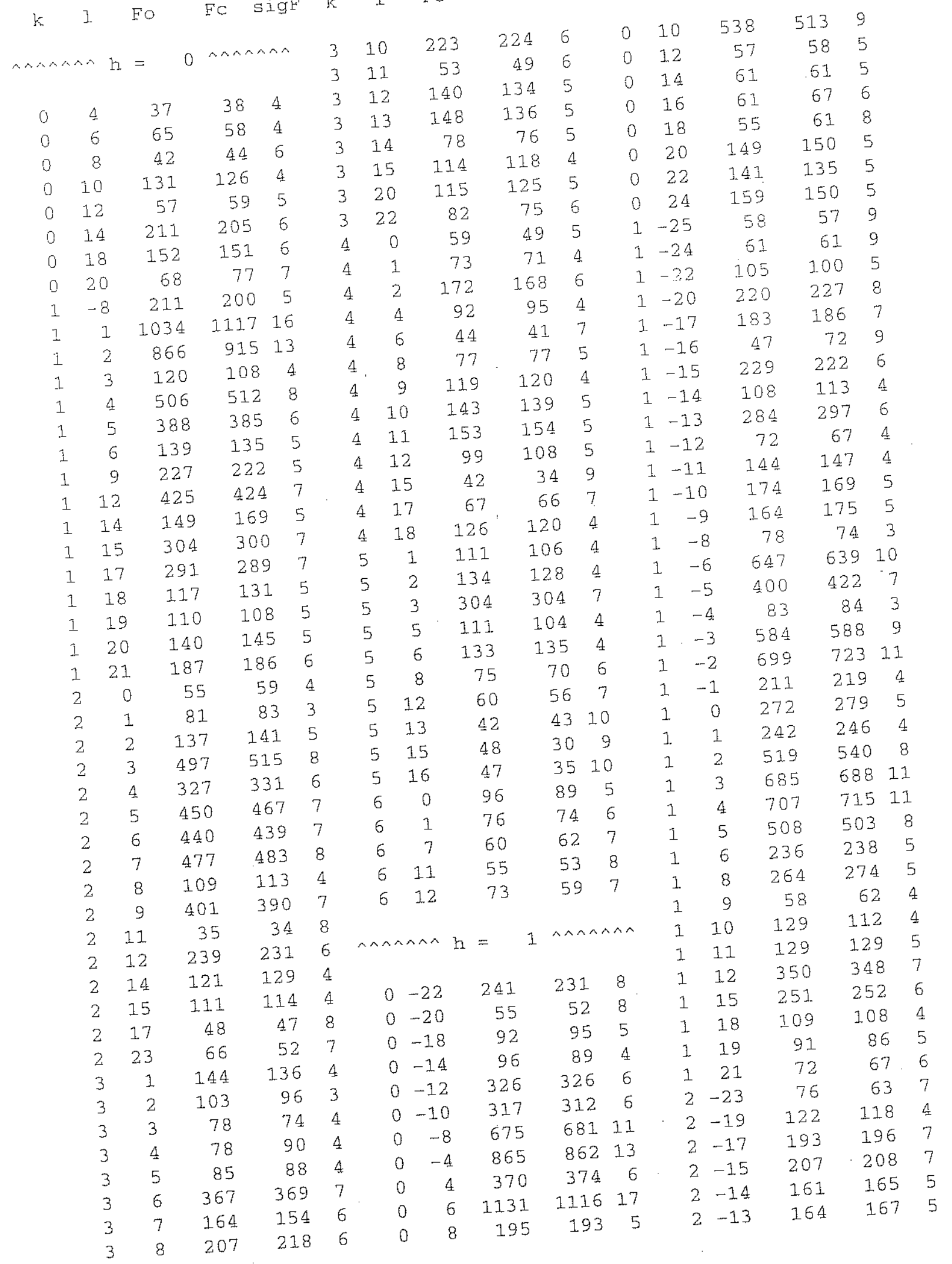


1.0|Flo vs $10 \mid \mathrm{FlC}$

page 2

\begin{tabular}{|c|c|c|c|c|c|c|c|c|c|c|c|c|c|}
\hline 1 & Fo & $\mathrm{FC}$ & $\operatorname{sig} F$ & k & 1 & EO & FC & $\operatorname{sigF}$ & $\mathrm{k}$ & 1 & Fo & $\mathrm{FC}$ & $\operatorname{sigF}$ \\
\hline $\begin{array}{ll}2 & -12\end{array}$ & 168 & 178 & 5 & 3 & 4 & 45 & 45 & 5 & 5 & -5 & 48 & 43 & 8 \\
\hline $\begin{array}{l}2-11 \\
2-10\end{array}$ & 64 & 67 & 5 & 3 & 5 & 161 & 167 & 5 & 5 & -1 & 203 & 197 & 6 \\
\hline-10 & 216 & 2.12 & 6 & 3 & 6 & 1.15 & 110 & 4 & 5 & 0 & 77 & 69 & 5 \\
\hline-9 & 1.58 & 151 & 6 & 3 & 7 & 74 & 72 & 4 & 5 & 1 & 254 & 157 & 5 \\
\hline-8 & 67 & 71 & 4 & 3 & 8 & 46 & 59 & 6 & 5 & 2 & 1.13 & 122 & 4 \\
\hline $\begin{array}{l}-7 \\
-6\end{array}$ & 127 & 132 & 4 & 3 & 9 & 69 & 67 & 4 & 5 & 3 & 217 & 2.17 & 7 \\
\hline $\begin{array}{l}-6 \\
-5\end{array}$ & 88 & 96 & 3 & 3 & 10 & 151 & 147 & 4 & 5 & $\Delta$ & 155 & 158 & 5 \\
\hline $\begin{array}{l}-5 \\
-4\end{array}$ & 1.92 & 208 & 5 & 3 & 11 & 86 & 90 & 4 & 5 & 5 & 248 & $\begin{array}{r}254 \\
57\end{array}$ & 7 \\
\hline $\begin{array}{l}-4 \\
-3\end{array}$ & 194 & 191 & 5 & 3 & 12 & 154 & 159 & 5 & 5 & 6 & $\begin{array}{l}64 \\
85\end{array}$ & $\begin{array}{l}57 \\
75\end{array}$ & $\begin{array}{l}6 \\
5\end{array}$ \\
\hline $\begin{array}{l}-3 \\
-2\end{array}$ & 253 & 265 & $\begin{array}{l}5 \\
5\end{array}$ & 3 & 14 & 183 & 193 & 7 & 5 & $\begin{array}{l}7 \\
8\end{array}$ & $\begin{array}{l}85 \\
68\end{array}$ & $\begin{array}{l}15 \\
63\end{array}$ & $\begin{array}{l}5 \\
6\end{array}$ \\
\hline $\begin{array}{l}-2 \\
-1\end{array}$ & 20 & $\begin{array}{l}203 \\
2.96\end{array}$ & $\begin{array}{l}5 \\
5\end{array}$ & 3 & 15 & 80 & 87 & 6 & $\begin{array}{l}5 \\
5\end{array}$ & $\begin{array}{r}8 \\
12\end{array}$ & 102 & 95 & $\begin{array}{l}6 \\
5\end{array}$ \\
\hline $\begin{array}{r}-1 \\
0\end{array}$ & $\begin{array}{r}282 \\
53\end{array}$ & $\begin{array}{r}296 \\
60\end{array}$ & $\begin{array}{l}5 \\
4\end{array}$ & 3 & $\begin{array}{l}16 \\
20\end{array}$ & $\begin{array}{r}220 \\
69\end{array}$ & $\begin{array}{r}223 \\
67\end{array}$ & 6 & 5 & 13 & 76 & 90 & 6 \\
\hline 1 & 60 & 65 & $\begin{array}{l}4 \\
4\end{array}$ & $\begin{array}{l}3 \\
3\end{array}$ & 22 & 71 & 70 & 7 & 6 & -3 & 146 & 145 & 5 \\
\hline 2 & 264 & 269 & 5 & $4-$ & -19 & 101 & 90 & 5 & 6 & -1 & 100 & 109 & 5 \\
\hline 3 & 226 & 231 & 5 & $4-$ & -18 & 68 & 74 & 7 & 6 & 0 & 134 & 130 & 5 \\
\hline 4 & 230 & 233 & 5 & $4-$ & -17 & 156 & 141 & 5 & 6 & 1 & 170 & 177 & 6 \\
\hline 5 & 51 & 60 & 5 & $4-$ & -15 & 81 & 79 & 5 & 6 & 3 & 65 & 67 & 7 \\
\hline 6 & 110 & 109 & 4 & 4 & -12 & 51 & 52 & 7 & & & & & \\
\hline 7 & 144 & 142 & 4 & 4 & -11 & 134 & 135 & 5 & & $\leadsto \mathrm{h}$ & $z=$ & 2 & \\
\hline 8 & 352 & 352 & 6 & 4 & -10 & 74 & 77 & 5 & & & 124 & & \\
\hline 9 & 158 & $\begin{array}{l}163 \\
256\end{array}$ & $\begin{array}{l}5 \\
6\end{array}$ & 4 & -9 & 251 & 260 & 6 & $\begin{array}{l}0 \\
0 .\end{array}$ & $\begin{array}{l}-20 \\
-18\end{array}$ & $\begin{array}{l}124 \\
135\end{array}$ & $\begin{array}{l}124 \\
127\end{array}$ & 4 \\
\hline 11. & 251 & $\begin{array}{l}256 \\
116\end{array}$ & $\begin{array}{l}6 \\
4\end{array}$ & 4 & -7 & 127 & 131 & 5 & $\begin{array}{l}0 \\
0\end{array}$ & $\begin{array}{l}-18 \\
-1.6\end{array}$ & 76 & 70 & $\begin{array}{l}4 \\
5\end{array}$ \\
\hline 12 & 12.5 & $\begin{array}{l}116 \\
254\end{array}$ & $\begin{array}{l}4 \\
6\end{array}$ & 4 & -6 & 41 & 35 & 8 & $\begin{array}{l}0 \\
0\end{array}$ & $\begin{array}{l}-10 \\
-12\end{array}$ & 438 & 427 & $\begin{array}{l}5 \\
8\end{array}$ \\
\hline 13 & 257 & $\begin{array}{l}254 \\
108\end{array}$ & $\begin{array}{l}6 \\
4\end{array}$ & $\Delta$ & -5 & 88 & 92 & 4 & $\begin{array}{l}0 \\
0\end{array}$ & -10 & 423 & 424 & $\begin{array}{l}8 \\
7\end{array}$ \\
\hline 15 & 110 & $\begin{array}{l}108 \\
157\end{array}$ & $\begin{array}{l}4 \\
6\end{array}$ & 4 & -4 & 116 & 122 & 4 & $\begin{array}{l}0 \\
0\end{array}$ & -8 & 637 & 6481 & 10 \\
\hline 17 & 158 & $\begin{array}{r}157 \\
82\end{array}$ & $\begin{array}{l}6 \\
6\end{array}$ & 4 & -3 & 81 & 90 & 4 & 0 & -6 & 1001 & 1017 & 15 \\
\hline 18 & $\begin{array}{r}75 \\
228\end{array}$ & $\begin{array}{r}82 \\
231\end{array}$ & $\begin{array}{l}6 \\
7\end{array}$ & 4 & -2 & $\begin{array}{r}92 \\
155\end{array}$ & $\begin{array}{r}91 \\
165\end{array}$ & $\begin{array}{l}4 \\
5\end{array}$ & 0 & -4 & 401 & 382 & 6 \\
\hline 19 & $\begin{array}{r}228 \\
76\end{array}$ & $\begin{array}{r}231 \\
78\end{array}$ & $\begin{array}{l}7 \\
6\end{array}$ & $\begin{array}{l}4 \\
4\end{array}$ & $\begin{array}{r}-1 \\
0\end{array}$ & $\begin{array}{l}155 \\
192\end{array}$ & $\begin{array}{l}165 \\
198\end{array}$ & 6 & 0 & -2 & 154 & 156 & 4 \\
\hline $\begin{array}{l}20 \\
21\end{array}$ & 65 & 81 & $\begin{array}{l}6 \\
7\end{array}$ & $\begin{array}{l}4 \\
4\end{array}$ & $\begin{array}{l}0 \\
1\end{array}$ & 123 & 129 & 4 & 0 & 0 & 44 & 41 & 3 \\
\hline $\begin{array}{rr}2 & 21 \\
3 & -22\end{array}$ & 73 & 70 & 7 & 4 & 2 & 191 & 199 & 6 & 0 & 2 & 305 & 301 & 5 \\
\hline $3-20$ & 80 & 85 & 6 & 4 & 4 & 42 & 37 & 7 & 0 & 4 & 2345 & 2330 & 36 \\
\hline $3-15$ & 79 & 85 & 5 & 4 & 5 & 129 & 130 & 4 & 0 & 6 & 412 & 396 & 7 \\
\hline $3-14$ & 46 & 34 & 8 & 4 & 6 & 59 & 40 & 5 & 0 & 8 & 196 & 192 & 5 \\
\hline $3-13$ & 121 & 117 & 4 & 4 & 8 & 265 & 275 & 6 & 0 & 10 & 325 & 335 & 6 \\
\hline $3-1.1$ & 58 & 55 & 6 & 4 & 9 & 81 & 86 & 5 & 0 & 12 & 225 & 214 & 5 \\
\hline $3-10$ & 54 & 50 & 5 & 4 & 11 & 71 & 70 & 5 & 0 & 16 & 316 & 312 & 7 \\
\hline $\begin{array}{ll}3 & -9\end{array}$ & 82 & 82 & 4 & 4 & 12 & 91 & 98 & 5 & 0 & 18 & 131 & 124 & 4 \\
\hline-8 & 115 & 122 & 4 & 4 & 14 & 69 & 56 & 6 & 0 & 20 & 198 & 191 & 5 \\
\hline-7 & 266 & 265 & 6 & $\Delta$ & 16 & 50 & 54 & 8 & 0 & 22 & 142 & 142 & 5 \\
\hline-6 & 180 & 195 & 5 & 4 & 17 & 78 & 70 & 6 & 1 & -2.2 & 108 & 123 & 5 \\
\hline-5 & 81 & 84 & 4 & 4 & 18 & 101 & 1.09 & 5 & 1 & -21 & 187 & 186 & 6 \\
\hline-4 & 398 & 401 & 7 & $5-$ & -16 & 67 & 65 & 7 & 1 & -17 & 191 & 185 & 7 \\
\hline $\begin{array}{l}-2 \\
-1\end{array}$ & 364 & 366 & 7 & $5-$ & -13 & 81 & 74 & 6 & 1 & -14 & 203 & $\begin{array}{r}208 \\
56\end{array}$ & 6 \\
\hline $\begin{array}{r}-1 \\
0\end{array}$ & 86 & 82 & 3 & 5 & -11 & 97 & 89 & 5 & 1 & -12 & $\begin{array}{l}62 \\
61\end{array}$ & $\begin{array}{l}56 \\
51\end{array}$ & 5 \\
\hline $\begin{array}{l}0 \\
1\end{array}$ & 207 & $\begin{array}{r}212 \\
63\end{array}$ & $\begin{array}{l}5 \\
4\end{array}$ & $5-$ & -10 & 61 & $\begin{array}{r}66 \\
107\end{array}$ & $\begin{array}{l}7 \\
4\end{array}$ & $\begin{array}{l}1 \\
1\end{array}$ & $\begin{array}{l}-11 \\
-10\end{array}$ & 55 & $\begin{array}{l}51 \\
57\end{array}$ & 5 \\
\hline $\begin{array}{l}1 \\
2\end{array}$ & $\begin{array}{r}65 \\
430\end{array}$ & $\begin{array}{r}03 \\
430\end{array}$ & $\begin{array}{l}4 \\
7\end{array}$ & $\begin{array}{l}5 \\
5\end{array}$ & $\begin{array}{l}-9 \\
-7\end{array}$ & $\begin{array}{l}116 \\
127\end{array}$ & $\begin{array}{l}107 \\
129\end{array}$ & 5 & 1 & -9 & 303 & 301 & \\
\hline & & 110 & 4 & 5 & -6 & 55 & 59 & 7 & 1 & -7 & 461 & 462 & \\
\hline
\end{tabular}


$10|F| O$ VS $10|\mathrm{~F}| \mathrm{C}$

page 3

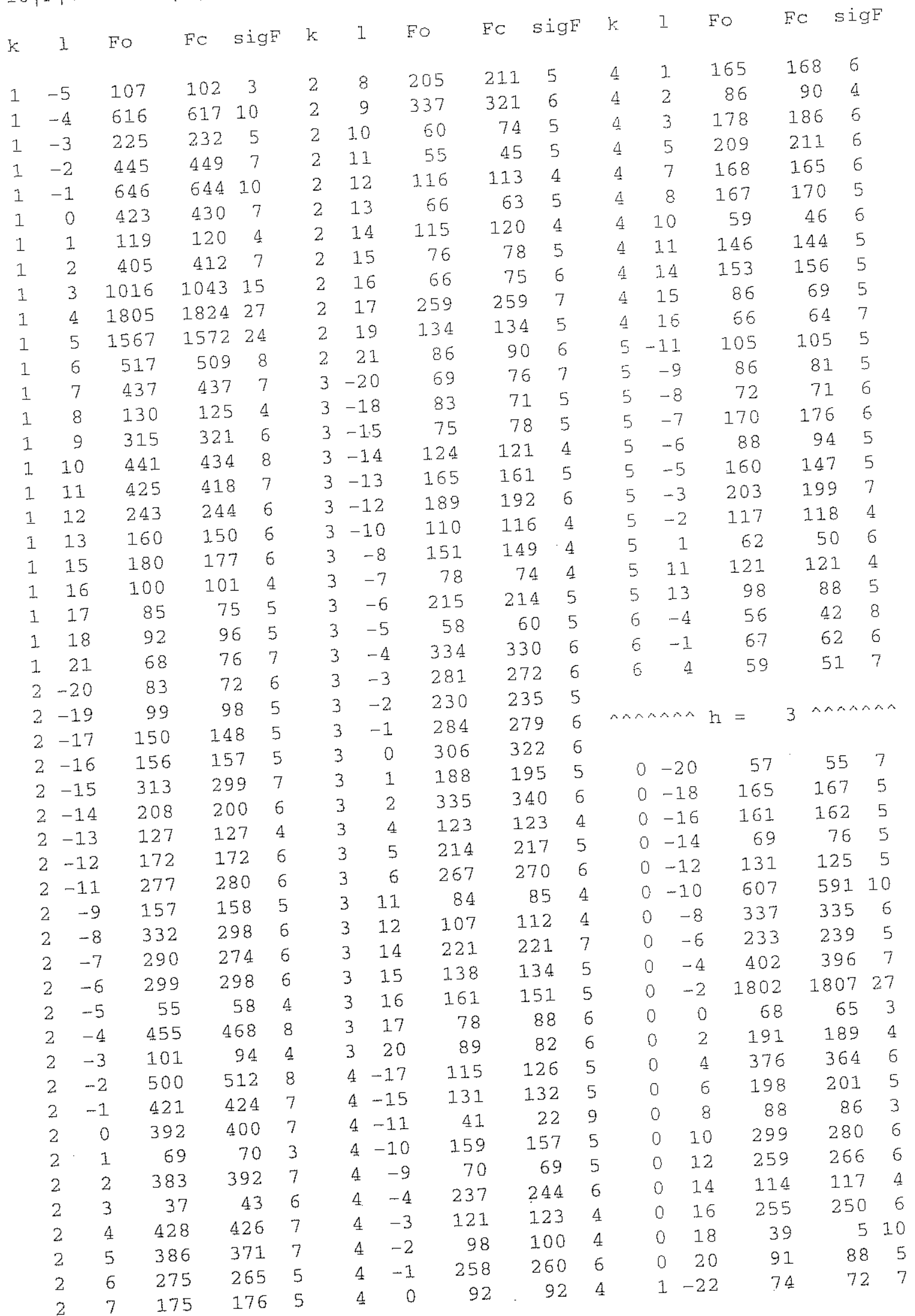


$10|F| O$ VS $10|F| C$

page 4

\begin{tabular}{|c|c|c|c|c|c|c|c|c|c|c|c|c|c|c|}
\hline & I. & FO & $\mathrm{FC}$ & $\operatorname{sig} F$ & k & 1 & Fo & $\mathrm{EC}$ & sigF & $k$ & 1 & Fo & $\mathrm{EC}$ & sigF \\
\hline$i$ & -21 & 70 & 76 & 7 & 2 & -5 & 465 & 465 & 8 & 3 & 16 & 67 & 69 & 6 \\
\hline & -20 & 49 & 47 & 8 & 2 & -4 & 59 & 65 & 4 & 4 & -16 & 73 & 70 & 6 \\
\hline 1. & -19 & 131 & 128 & 5 & 2 & -3 & 117 & 122 & 4 & 4 & -14 & 45 & 23 & 9 \\
\hline & -18 & 129 & 132 & 5 & 2 & -2 & 258 & 245 & 5 & 4 & -9 & 90 & 92 & 4 \\
\hline 2 & -17 & 194 & 183 & 7 & 2 & -1 & 247 & 244 & 5 & 4 & -8 & 40 & 21 & 8 \\
\hline $\mathbb{1}$ & -15 & 91 & 91. & 4 & 2 & 0 & 149 & 153 & 5 & 4 & -7 & 170 & 167 & 5 \\
\hline 1 & -13 & 211 & 214 & 6 & 2 & 1 & 375 & 370 & 7 & 4 & -6 & 249 & 249 & 6 \\
\hline 1 & -12 & 272 & 266 & 6 & 2 & 2 & 354 & 352 & 6 & 4 & -5 & 233 & 238 & 6 \\
\hline 1 & -11 & 436 & 420 & 8 & 2 & 3 & 21.5 & 227 & 5 & 4 & -4 & 155 & 145 & 5 \\
\hline 1 & -10 & 92 & 95 & 3 & 2 & 4 & 142 & 155 & 5 & 4 & -2 & 75 & 72 & 4 \\
\hline 1 & -9 & 109 & 103 & 4 & 2 & 5 & 156 & 159 & 5 & 4 & 0 & 174 & 183 & 5 \\
\hline 1 & -8 & 198 & 190 & 5 & 2 & 6 & 65 & 60 & 4 & 4 & 2 & 229 & 232 & 6 \\
\hline 1 & -7 & 83 & 82 & 3 & 2 & 7 & 525 & 524 & 9 & 4 & 3 & 75 & 84 & 5 \\
\hline 1 & -6 & 46 & 50 & 5 & 2 & 8 & 293 & 309 & 6 & 4 & 4 & 120 & 120 & 4 \\
\hline 1 & -4 & 553 & 569 & 9 & 2 & 9 & 454 & 451 & 8 & 4 & 5 & 164 & 163 & 5 \\
\hline 1 & -3 & 1143 & 1156 & 17 & 2 & 10 & 65 & 67 & 5 & 4 & 7 & 257 & 259 & 6 \\
\hline 1 & -2 & 153 & 161 & 4 & 2 & 13 & 71 & 71 & 5 & 4 & 8 & 199 & 198 & 6 \\
\hline 1 & -1 & 146 & 140 & 4 & 2 & 14 & 50 & 51 & 7 & 4 & 9 & 245 & 250 & 7 \\
\hline 1 & 0 & 382 & 385 & 6 & 2 & 16 & 71 & 75 & 6 & $\Delta$ & 10 & 104 & 108 & 4 \\
\hline 1 & 1 & 507 & 508 & 8 & 2 & 18 & 173 & 170 & 5 & 4 & 16 & 117 & 111 & 5 \\
\hline 1 & 2 & 78 & 73 & 3 & 2 & 19 & 11.0 & 105 & 5 & 5 & -12 & 89 & 91 & 5 \\
\hline 1 & 3 & 258 & 263 & 5 & 2 & 20 & 68 & 65 & 7 & 5 & -10 & 108 & 105 & 5 \\
\hline 1 & 4 & 281 & 275 & 5 & 2 & 21 & 62 & 51 & 7 & 5 & -7 & 108 & 101 & 4 \\
\hline 1 & 5 & 136 & 139 & 5 & 3 & -19 & 48 & 42 & 9 & 5 & -5 & 236 & 238 & 7 \\
\hline 1 & 6 & 49 & 54 & 5 & 3 & -14 & 166 & 174 & 5 & 5 & -4 & .72 & 69 & 6 \\
\hline 1 & 7 & 64 & 66 & 4 & 3 & -12 & 297 & 294 & 7 & 5 & -3 & 54 & 54 & 7 \\
\hline$I$ & 8 & 313 & 292 & 6 & 3 & -11 & 65 & 53 & 5 & 5 & -1 & 77 & 71 & 5 \\
\hline 1 & 9 & 205 & 201 & 5 & 3 & -10 & 187 & 194 & 6 & 5 & 0 & 125 & 125 & 4 \\
\hline 1 & 10 & 316 & 325 & 6 & 3 & -9 & 101 & 100 & 4 & 5 & 1 & 112 & 106 & $\Delta$ \\
\hline 1 & 11. & 212 & 220 & 6 & 3 & -8 & 303 & 317 & 6 & 5 & 2 & 47 & 47 & 8 \\
\hline 1 & 12 & 158 & 152 & 4 & 3 & -7 & 163 & 173 & 5 & 5 & 4 & 54 & 39 & 7 \\
\hline 1 & 13 & 253 & 247 & 6 & 3 & -6 & 63 & 76 & 5 & 5 & 5 & 41 & 52 & 9 \\
\hline 1 & 15 & 234 & 233 & 6 & 3 & -5 & 153 & 146 & 5 & 5 & 6 & 87 & 86 & 5 \\
\hline 1 & 16 & 102 & 99 & 4 & 3 & -4 & 221 & 215 & 5 & 5 & 7 & 111 & 119 & 4 \\
\hline 1 & 17 & 60 & 54 & 7 & 3 & -3 & 187 & 176 & 5 & 5 & 8 & 70 & 79 & 6 \\
\hline 1 & 18 & 119 & 117 & 4 & 3 & -2 & 85 & 84 & 4 & 5 & 10 & 67 & 59 & 6 \\
\hline 1 & 19 & 177 & 1.75 & 5 & 3 & -1 & 60 & 57 & 4 & 5 & 11 & 102 & 96 & 5 \\
\hline 1 & 22 & 83 & 80 & 6 & 3 & 0 & 21.2 & 199 & 5 & 5 & 12 & 58 & 55 & 7 \\
\hline 2 & -21 & 89 & 85 & 6 & 3 & 1 & 108 & 104 & 4 & 6 & -2 & 123 & 110 & 5 \\
\hline 2 & -20 & 131 & 135 & 4 & 3 & 2 & 93 & 100 & 3 & 6 & 0 & 91 & 82 & 5 \\
\hline 2 & -17 & 211 & 218 & 7 & 3 & 3 & 209 & 211 & 5 & 6 & 1 & 95 & 101 & 5 \\
\hline 2 & -15 & 203 & 197 & 7 & 3 & 4 & 382 & 392 & 7 & & & & & \\
\hline 2 & -14 & 75 & 85 & 5 & 3 & 5 & 41 & 31 & 6 & & $\operatorname{An} \mathrm{h}$ & $=$ & $\wedge \wedge n$ & nn \\
\hline 2 & -12 & 98 & 94 & 4 & 3 & 6 & 185 & 187 & 6 & & & & & \\
\hline 2 & -11 & 204 & 206 & 6 & 3 & 7 & 72 & 76 & 4 & 0 & -22 & 105 & 98 & 5 \\
\hline 2 & -10 & 141 & 143 & 5 & 3 & 9 & 37 & 32 & 8 & 0 & -1.8 & 364 & 353 & 8 \\
\hline 2 & -9 & 321 & 331 & 6 & 3 & 11 & 49 & 45 & 7 & 0 & -16 & 261 & 255 & 6 \\
\hline 2 & -8 & 66 & 60 & 4 & 3 & 12 & 213 & 216 & 6 & 0 & -14 & 178 & 174 & 6 \\
\hline 2 & -7 & 62 & 49 & 4 & 3 & 14 & 69 & 69 & 6 & 0 & -12 & 178 & 192 & 6 \\
\hline 2 & -6 & 33 & 30 & 7 & 3 & 15 & 55 & 58 & 7 & 0 & -10 & 354 & 368 & 6 \\
\hline
\end{tabular}


$10|\mathrm{~F}| O \mathrm{VS} \quad 10|\mathrm{~F}| \mathrm{C}$

page 5

$k 1$ Fo FC sigF k 1 Fo FC sigF k 1 Fo FC sigF

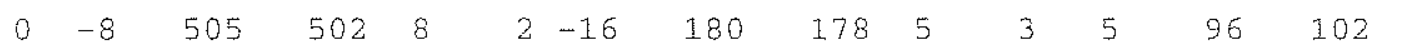

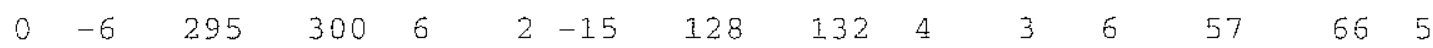

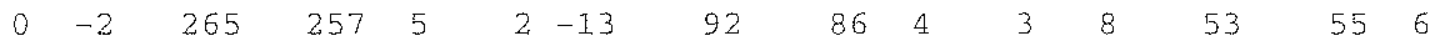

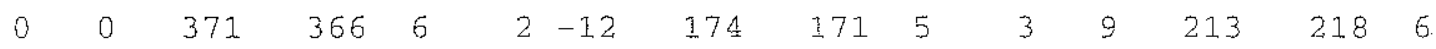

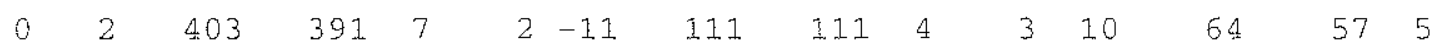

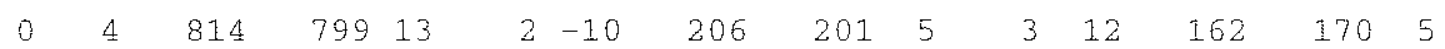

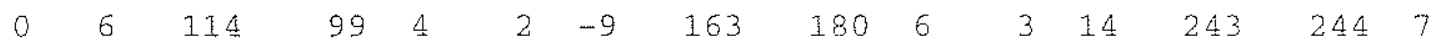

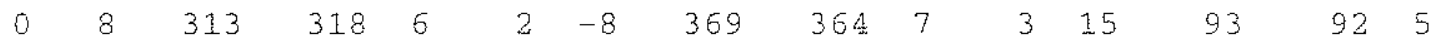

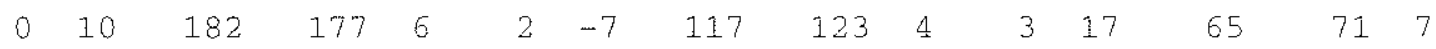

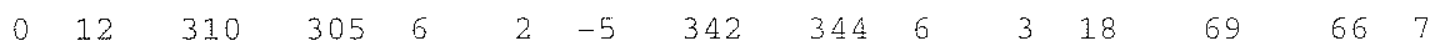

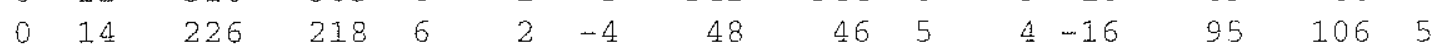

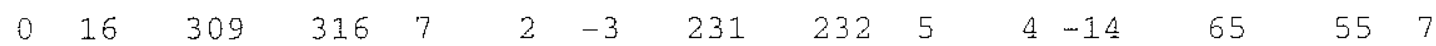

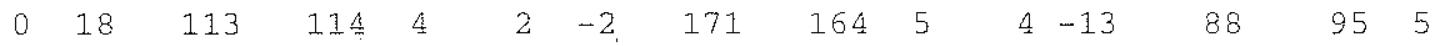

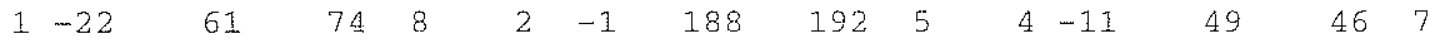

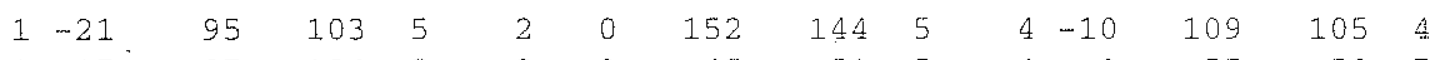

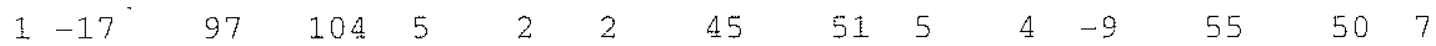

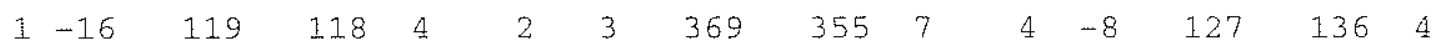

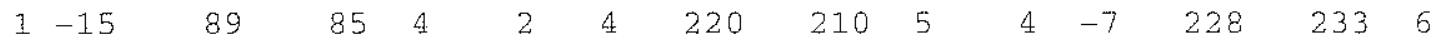

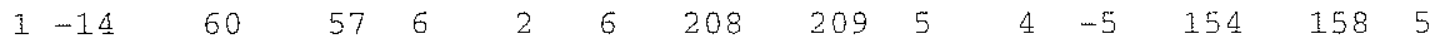

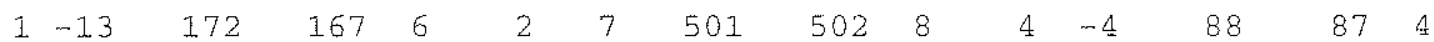

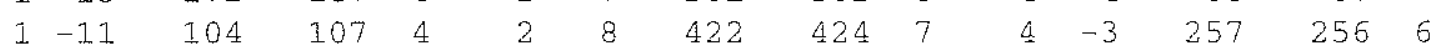

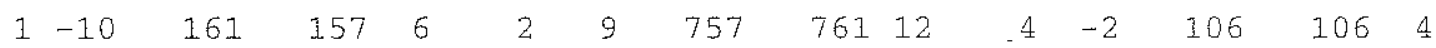

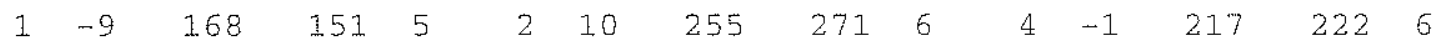

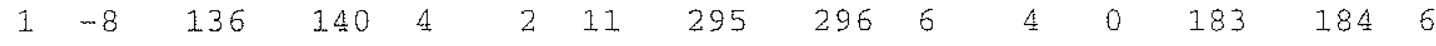

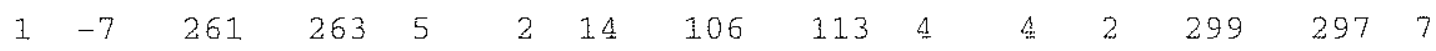

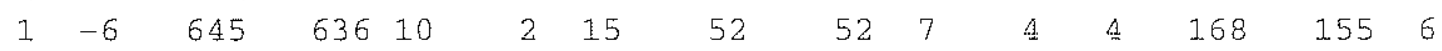

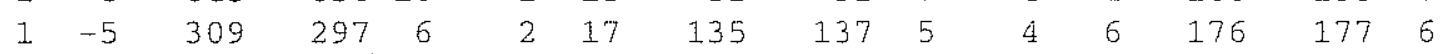

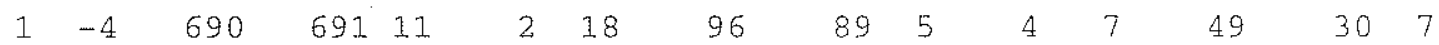

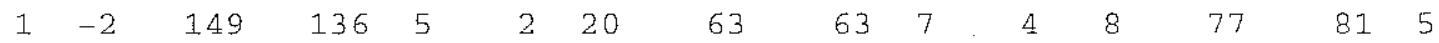

$\begin{array}{lllllllllllllll}1 & -1 & 414 & 414 & 7 & 3 & -19 & 81 & 93 & 6 & 4 & 12 & 89 & 97 & 5\end{array}$

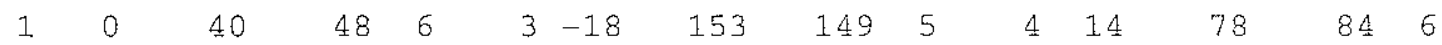

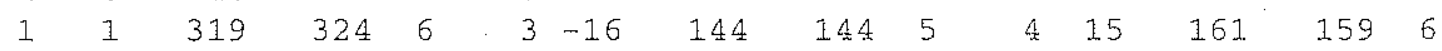

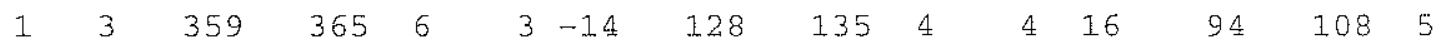

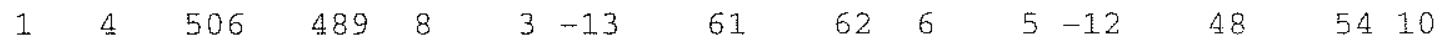

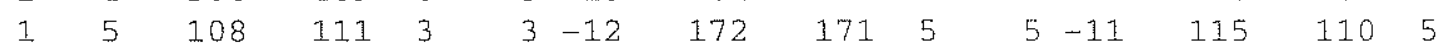

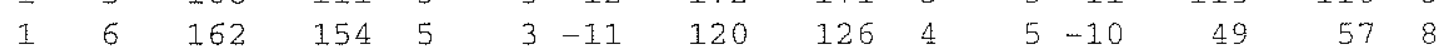

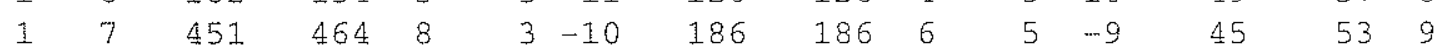

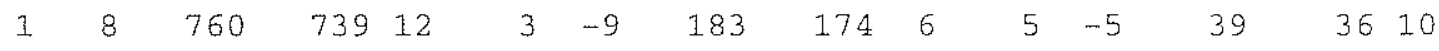

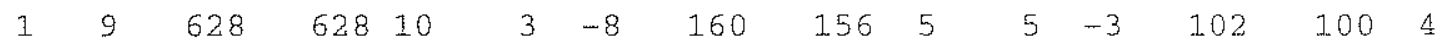

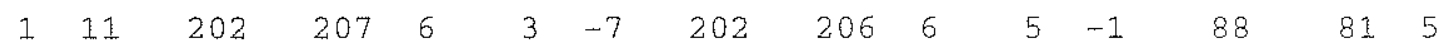

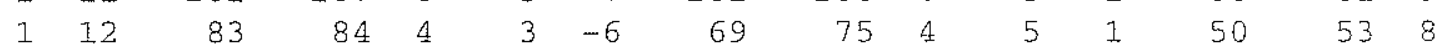

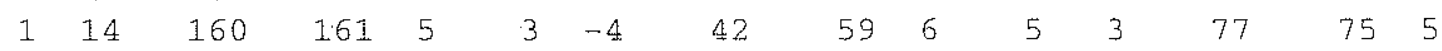

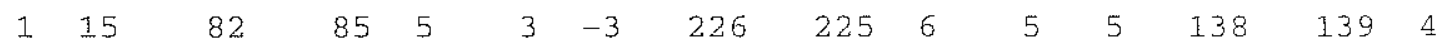

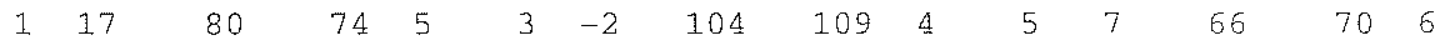

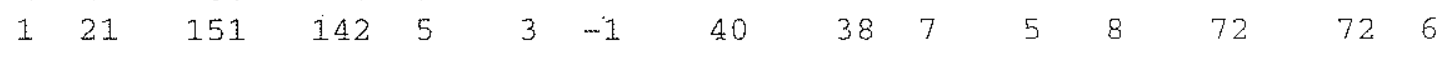

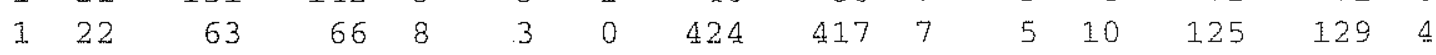

$\begin{array}{lllllllll}2 & -21 & 135 & 131 & 5 & 3 & 1 & 320 & 314\end{array}$

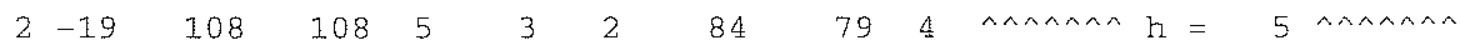


$10|\mathrm{~F}| \mathrm{O}$ VS $10|\mathrm{E}| \mathrm{C}$

page 6

$k 1 \mathrm{FO} F \mathrm{FC} \operatorname{sigF} k 1$ Fo FC sigF k 1 Fo FC sigF

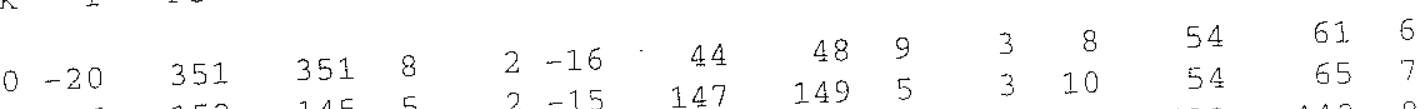

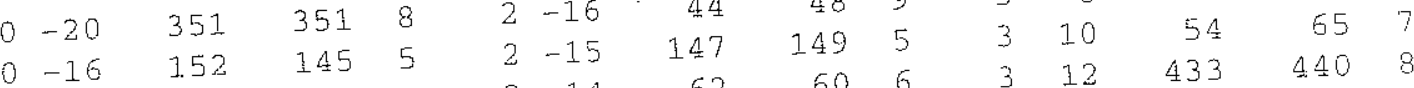

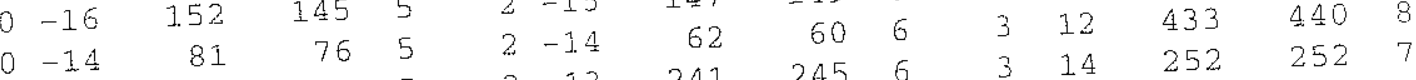

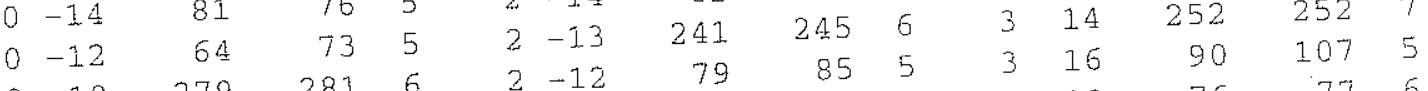

$\begin{array}{rrrrrrrrrrr}0 & -10 & 279 & 281 & 6 & 2 & -12 & 79 & 85 & 5 & 3\end{array}$

$\begin{array}{lllllllllllll}0 & -6 & 507 & 504 & 8 & 2 & -10 & 204 & 207 & 4 & 106 & 99 & 4\end{array}$

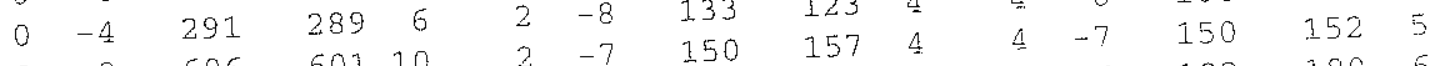

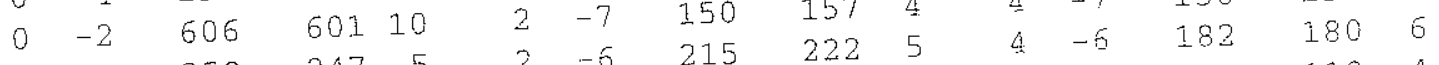

$\begin{array}{lllllllllllllll}0 & 2 & 258 & 247 & 5 & 2 & -6 & 215 & -5 & 112 & 119 & 4 \\ 0 & 6 & 387 & 391 & 7 & 2 & -5 & 170 & 168 & 5 & 4 & -3 & 254 & 253 & 6\end{array}$

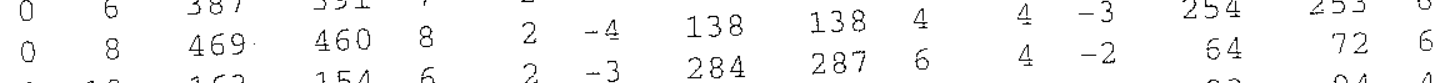

$\begin{array}{llllllllllllll}0 & 10 & 163 & 154 & 6 & 2 & -3 & 284 & 287 & 64 & 4 & 4 & 4 & 4\end{array}$

$\begin{array}{llllllrrrrrrr}0 & 14 & 44 & 45 & 8 & 2 & -2 & 235 & 215 & 5 & 4 & 6\end{array}$

$\begin{array}{rrrrrrrr}0 & 16 & 62 & 47 & 6 & 2 & -1 & 77 \\ 0 & 20 & 114 & 116 & 5 & 2 & 0 & 502\end{array}$

$\begin{array}{rrrrr}0 & 20 & 114 & 116 & 5 \\ 1 & -21 & 100 & 105 & 5\end{array}$

$1-18 \quad 142$

$1-15 \quad 73$

$\begin{array}{lll}305 & 315 & 6\end{array}$

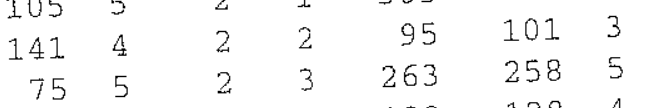

$1-13 \quad 151$

$149 \quad 5$

129

$\begin{array}{ll}258 & 5 \\ 128 & 4\end{array}$

$257 \quad 258 \quad 6$

$106 \quad 104 \quad 4$

$\begin{array}{rrrrr}1 & -11 & 144 & 144 & 5 \\ 1 & -10 & 63 & 72 & 5\end{array}$

$\begin{array}{lll}77 & 73 & 4\end{array}$

$1 \quad-9 \quad 259$

2836

$\begin{array}{ll}73 & 4 \\ 92 & 4\end{array}$

$\begin{array}{ll}117 & 4 \\ 305 & 6\end{array}$

95

$-8 \quad 116$

$\begin{array}{ll}-7 & 321\end{array}$

$1 \quad-6 \quad 627$

627.10

537

$\begin{array}{rrr}47 & 328 & 7\end{array}$

2335

$\begin{array}{lll}321 & 328 & 7 \\ 146 & 147 & 5 \\ 203 & 205 & 6\end{array}$

$205200 \quad 6$

$\begin{array}{lllll}1 & -5 & 231 & 233 & 5 \\ 1 & -3 & 218 & 223 & 5\end{array}$

$\begin{array}{rrrrr}1 & -2 & 180 & 180 & 5 \\ 1 & -1 & 58 & 55 & 4\end{array}$

$223 \quad 241 \quad 7$

$\begin{array}{lll}159 & 160 & 5\end{array}$

$\begin{array}{rr}55 & 4 \\ 287\end{array}$

65

627

417

$407 \quad 7$

99

1.02 .5

$\begin{array}{lllllll}415 & 7 & 3 & -17 & 103 & 104 & 5 \\ 296 & 6 & 3 & -16 & 119 & 114 & 4\end{array}$

294

$296 \quad 6$

362

3496

$3-14$

114

1204

1015

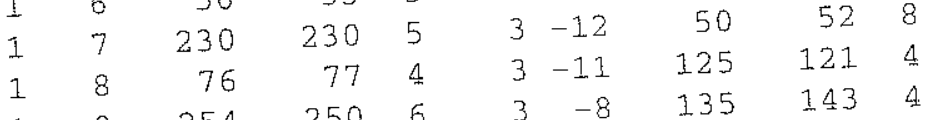

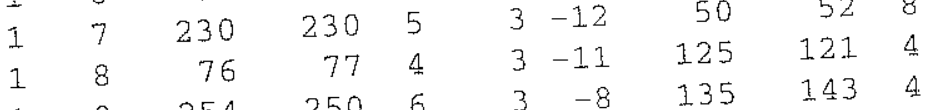

$3-13$

95

528

$\begin{array}{rrrrrrrrrr}1 & 7 & 230 & 230 & 5 & 3 & -12 & 50 & 52 & 8 \\ 1 & 8 & 76 & 77 & 4 & 3 & -11 & 125 & 121 & 4 \\ 1 & 9 & 254 & 250 & 6 & 3 & -8 & 135 & 143 & 4\end{array}$

43

80

805

$\begin{array}{lllll}4 & 4 & 80 & 80 & 5\end{array}$

$4972 \quad 735$

$4 \quad 10 \quad 170 \quad 1745$

$\begin{array}{lllll}4 & 11 & 118 & 132 & 4\end{array}$

$\begin{array}{lllll}4 & 12 & 133 & 145 & 4\end{array}$

$4 \quad 14 \quad 70 \quad 74 \quad 6$

$\begin{array}{lllll}5 & -8 & 59 & 51 & 7\end{array}$

$\begin{array}{lllll}5 & -7 & 92 & 79 & 5\end{array}$

$\begin{array}{lllll}5 & -6 & 85 & 89 & 5\end{array}$

$\begin{array}{lllll}5 & -5 & 195 & 187 & 7\end{array}$

$\begin{array}{lllll}5 & -4 & 100 & 99 & 5\end{array}$

$\begin{array}{lllll}5 & -3 & 117 & 112 & 4\end{array}$

$\begin{array}{llllllllll}1 & 9 & 254 & 250 & 6 & 3 & -8 & 135 & 143 & 4 \\ 1 & 10 & 135 & 126 & 5 & 3 & -7 & 123 & 121 & 4\end{array}$

$\begin{array}{rrrrrrrrrr}1 & 10 & 135 & 126 & 5 & 3 & -7 & 123 & 121 & 4 \\ 1 & 11 & 160 & 159 & 6 & 3 & -5 & 53 & 53 & 5 \\ 1 & 12 & 57 & 55 & 6 & 3 & -4 & 170 & 179 & 6\end{array}$

$\wedge$ ค ^ $\mathrm{h}=6$

$\wedge \wedge \wedge \wedge \wedge \wedge$

$\begin{array}{rrrrrrrrrr}1 & 12 & 57 & 55 & 6 & 3 & -4 & 170 & 179 & 6 \\ 1 & 13 & 242 & 235 & 6 & 3 & -3 & 224 & 221 & 6\end{array}$

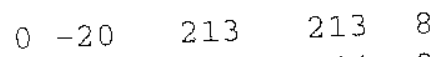

$\begin{array}{lllll}0 & -16 & 43 & 44 & 9\end{array}$

$\begin{array}{lllll}0 & -14 & 347 & 342 & 7\end{array}$

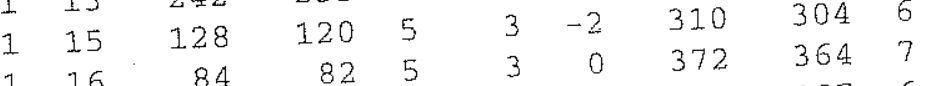

$\begin{array}{lllll}0 & -12 & 133 & 131 & 5\end{array}$

116

84

825

3646
1876

$\begin{array}{lll}0 & -8 & 40\end{array}$

$47 \quad 7$

118

51

705

188

1696

$\begin{array}{lll}0 & -6 & 198\end{array}$

1825

$\begin{array}{rrr}1 & 21 & 51 \\ 2 & -20 & 94\end{array}$

579

161

126.4

773

$762 \quad 12$

$2-18 \quad 61$

647
123

174

1796
774

$0 \quad 0 \quad 617$

61810

$\begin{array}{ll}2 & -17\end{array}$ 1.23 123 84

$\begin{array}{lll}0 & 2 & 81 \\ 0 & 4 & 77\end{array}$

$\begin{array}{ll}79 & 3\end{array}$ 
$10|\mathrm{~F}| O$ VS $10|\mathrm{~F}| \mathrm{C}$

$k 1$ FO FC sigF $k 1$ Fo FC sigF $k 1$ Fo 1 FC sigF

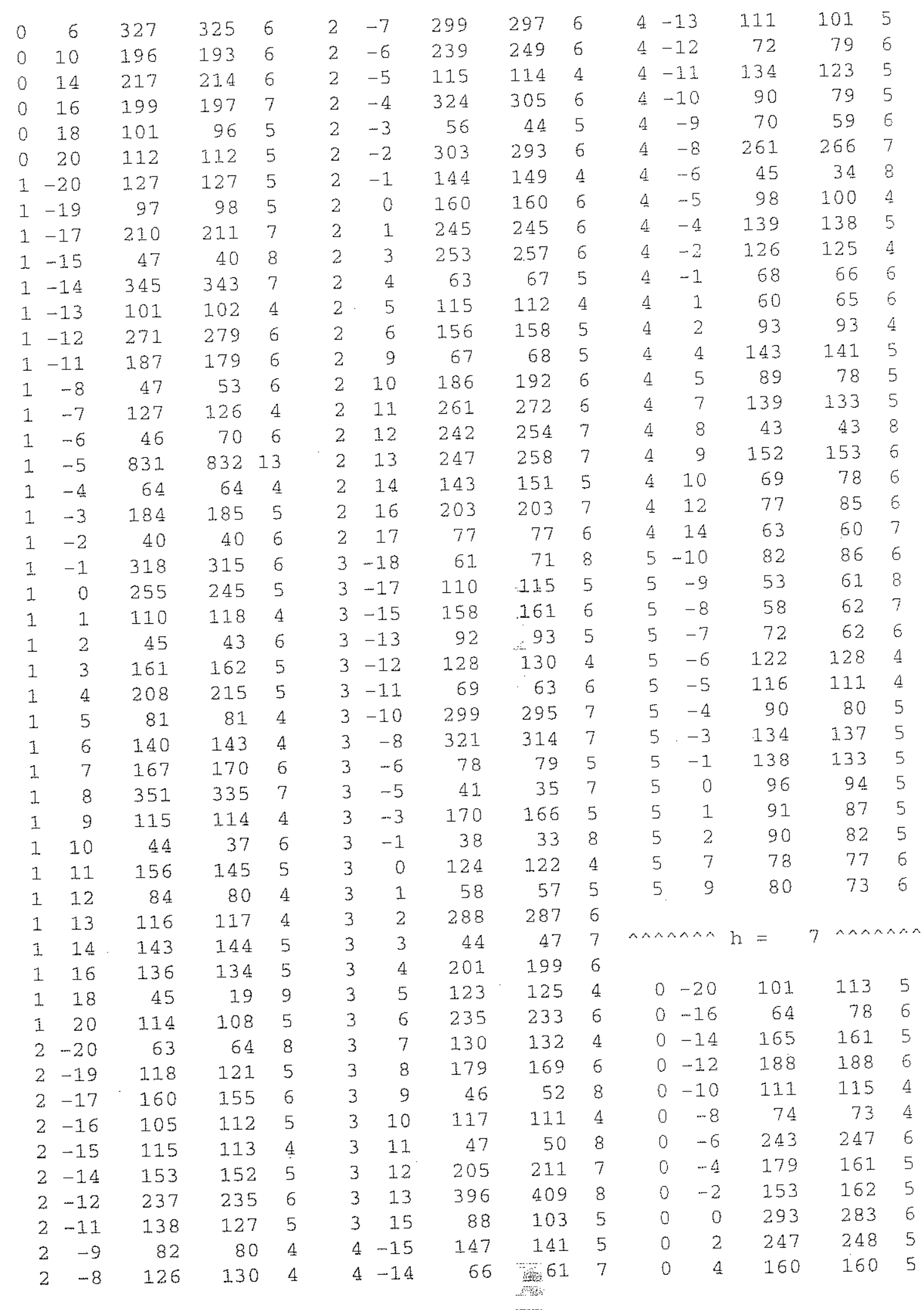




\begin{tabular}{|c|c|c|c|c|c|c|c|c|c|c|c|c|c|c|}
\hline k & 1 & Fo & $\mathrm{FC}$ & $s i g F$ & $\mathrm{k}$ & 1 & Fo & EC & Sigf' & $\mathrm{k}$ & 1 & EO & $\mathrm{FC}$ & $5 i g F$ \\
\hline 0 & 6 & 574 & 557 & 9 & 2 & 2 & 132 & 137 & 5 & 4 & -1 & 148 & 138 & 5 \\
\hline 0 & 8 & 69 & 72 & 5 & 2 & 3 & 184 & 174 & 6 & 4 & 0 & 187 & 194 & 5 \\
\hline 0 & 10 & 123 & 126 & $\Delta$ & 2 & 4 & 302 & 282 & 6 & 4 & 1 & 155 & 162 & 5 \\
\hline 0 & 14 & 243 & 238 & 7 & 2 & 5 & 165 & 1.66 & 5 & 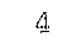 & 2 & 119 & 124 & 4 \\
\hline 0 & 16 & 107 & 97 & 5 & 2 & 6 & 184 & 187 & 6 & 4 & 3 & 46 & 48 & 8 \\
\hline 0 & 18 & 150 & 150 & 5 & 2 & 7 & 165 & 1.68 & 5 & 4 & 4 & 91 & 101 & 5 \\
\hline 0 & 20 & 94 & 93 & 5 & 2 & 8 & 104 & 107 & 4 & 4 & 5 & 67 & 64 & 6 \\
\hline 1 & -17 & 237 & 240 & 7 & 2 & 9 & 151 & 148 & 5 & 4 & 8 & 83 & 81 & 5 \\
\hline 1 & -16 & 143 & 1.43 & 5 & 2 & 10 & 73 & 75 & 5 & 4 & 9 & 60 & 68 & 7 \\
\hline 1 & -15 & 266 & 264 & 7 & 2 & 11 & 274 & 274 & 7 & 4 & 10 & 40 & 52 & 10 \\
\hline 1 & -14 & 57 & 57 & 7 & 2 & 12 & 111 & 1.19 & 4 & 4 & 11 & 95 & 86 & 5 \\
\hline 1 & -12 & 132 & 132 & 5 & 2 & 13 & 40 & 39 & 1.0 & 4 & 13 & 60 & 58 & 7 \\
\hline 1 & -11 & 145 & 148 & 5 & 2 & 14 & 94 & 95 & 5 & 5 & -8 & 58 & 66 & 8 \\
\hline 1 & -9 & 324 & 327 & 7 & 2 & 16 & 98 & 92 & 5 & 5 & -5 & 72 & 64 & 6 \\
\hline 1 & -8 & 272 & 272 & 6 & 2 & 17 & 71 & 69 & 6 & 5 & -3 & 98 & 90 & 5 \\
\hline I & -7 & 135 & 137 & 5 & 2 & 18 & 1.01 & 106 & 5 & 5 & -2 & 61 & 70 & 7 \\
\hline 1 & -6 & 212 & 213 & 6 & 3 & -13 & 66 & 67 & 6 & 5 & -1 & 82 & 64 & 5 \\
\hline 1 & -3 & 492 & 483 & 8 & 3 & -12 & 60 & 55 & 6 & 5 & 0 & 81 & 86 & 6 \\
\hline 1 & -2 & 206 & 199 & 5 & 3 & -11 & 62 & 65 & 6 & 5 & 3 & 99 & 95 & 5 \\
\hline 1 & -1 & 184 & 188 & 5 & 3 & -10 & 144 & 138 & 5 & 5 & 6 & 56 & 55 & 7 \\
\hline 1 & 0 & 281 & 272 & 6 & 3 & -9 & 262 & 263 & 7 & 5 & 8 & 73 & 67 & 6 \\
\hline 1 & 1 & 204 & 216 & 5 & 3 & -7 & 138 & 141 & 5 & & & & & \\
\hline 1 & 2 & 170 & 159 & 5 & 3 & -6 & 238 & 239 & 6 & $\mu n n$ & mn h & $=$ & & \\
\hline 1 & 4 & 162 & 152 & 5 & 3 & -5 & 97 & 99 & 4 & & & & & \\
\hline 1 & 5 & 56 & 72 & 5 & 3 & -4 & 183 & 188 & 6 & 0 & -16 & 206 & 209 & 7 \\
\hline 1 & 6 & 74 & 65 & 4 & 3 & -3 & 85 & 84 & 4 & 0 & -1.4 & 85 & 94 & 5 \\
\hline 1 & 7 & 87 & 86 & 4 & 3 & -2 & 127 & 127 & 5 & 0 & -12 & 288 & 288 & 7 \\
\hline 1 & 8 & 222 & 219 & 6 & 3 & -1 & 148 & 137 & 5 & 0 & -10 & 204 & 208 & 6 \\
\hline 1 & 9 & 178 & 181 & 5 & 3 & 1 & 85 & 89 & 4 & 0 & -8 & 323 & 3.19 & 6 \\
\hline 1 & 10 & 141 & 139 & 5 & 3 & 4 & 76 & 72 & 5 & 0 & -4 & 68 & 7.1 & 5 \\
\hline 1 & 11 & 61 & 68 & 6 & 3 & 5 & 114 & 119 & 4 & 0 & -2 & 178 & 168 & 6 \\
\hline 1 & 13 & 57 & 62 & 7 & 3 & 6 & 153 & 149 & 5 & 0 & 0 & 576 & 554 & 9 \\
\hline 1 & 18 & 81 & 79 & 6 & 3 & 8 & 118 & 116 & 4 & 0 & 2 & 55 & 52 & 5 \\
\hline 1 & 19 & 45 & 43 & 10 & 3 & 9 & 156 & 159 & 5 & 0 & 4 & 178 & 179 & 6 \\
\hline 1 & 20 & 75 & 75 & 6 & 3 & 10 & 74 & 77 & 5 & 0 & 6 & 203 & 205 & 6 \\
\hline 2 & -18 & 109 & 106 & 5 & 3 & 11 & 97 & 95 & 5 & 0 & 10 & 2.22 & 214 & 6 \\
\hline 2 & -15 & 52 & 58 & 8 & 3 & 12 & 156 & 160 & 5 & 0 & 12 & 252 & 248 & 6 \\
\hline 2 & -14 & 73 & 79 & 6 & 3 & 14 & 12.1 & 126 & 4 & 0 & 14 & 56 & 63 & 7 \\
\hline 2 & -10 & 84 & 81 & 5 & 3 & 16 & 191 & 205 & 6 & 0 & 16 & 107 & 109 & 5 \\
\hline 2 & -9 & 198 & 197 & 6 & 4 & -14 & 87 & 8.1 & 6 & 0 & 18 & 118 & 122 & 5 \\
\hline 2 & -8 & 68 & 63 & 5 & 4 & -12 & 161 & 158 & 5 & 1 & -17 & 102 & 109 & 5 \\
\hline 2 &.--7 & 242 & 242 & 6 & 4 & -11 & 115 & 118 & 5 & 1 & -15 & 149 & 154 & 5 \\
\hline 2 & -6 & 299 & 302 & 6 & 4 & -10 & 134 & 141 & 5 & 1 & -14 & 68 & 76 & 6 \\
\hline 2 & -5 & 87 & 89 & 4 & 4 & -9 & 219 & 219 & 7 & 1 & -12 & 136 & 139 & 5 \\
\hline 2 & -4 & 128 & 122 & 5 & 4 & -8 & 106 & 105 & 5 & 1 & -11 & 271 & 273 & 7 \\
\hline 2 & -3 & 191 & 185 & 6 & 4 & -6 & 104 & 106 & 5 & 1 & -10 & 1.22 & 124 & 4 \\
\hline 2 & -2 & 63 & 57 & 5 & 4 & -5 & 55 & 58 & 7 & 1 & -9 & 184 & 189 & 6 \\
\hline 2 & -1 & 87 & 81 & 4 & 4 & -4 & 44 & 54 & 9 & 1 & -8 & 136 & 135 & 5 \\
\hline 2 & 0 & 137 & 136 & 5 & 4 & -3 & 261 & 252 & 7 & 1 & -7 & 226 & 224 & 6 \\
\hline 2 & 1 & 61 & 59 & 5 & 4 & -2 & 99 & 102 & 4 & 1 & -5 & 41 & 53 & 7 \\
\hline
\end{tabular}




\begin{tabular}{|c|c|c|c|c|c|c|c|c|c|c|c|c|c|c|}
\hline k & 1 & Fo & $\mathrm{FC}$ & sigF & $k$ & 1 & Fo & FC & sigF & $k$ & 1 & Fo & $\mathrm{FC}$ & sigf \\
\hline 1. & -4 & 89 & 87 & 4 & 3 & -16 & 106 & 101 & 5 & 0 & -14 & 159 & 167 & 5 \\
\hline 1 & -2 & 161 & 153 & 6 & 3 & -13 & 98 & 100 & 5 & 0 & $-\cdots 12$ & 112 & 112 & 4 \\
\hline 1 & -1 & 332 & 308 & 6 & 3 & -12 & 156 & 166 & 6. & 0 & -10 & 119 & 117 & 4 \\
\hline 1 & 0 & 90 & 88 & 4 & 3 & -11 & 107 & 95 & 4 & 0 & -8 & 201 & 20.3 & 6 \\
\hline 1 & 1 & 230 & 216 & 6 & 3 & -10 & 65 & 56 & 6 & 0 & -6 & 160 & 165 & 5 \\
\hline 1 & 2 & 278 & 278 & 6 & 3 & -9 & 126 & 127 & 5 & 0 & -4 & 565 & 553 & 9 \\
\hline 1 & 3 & 282 & 286 & 6 & 3 & -8 & 180 & 176 & 5 & 0 & -2 & 133 & 134 & 4 \\
\hline 1 & 4 & 297 & 298 & 6 & 3 & -7 & 203 & 212 & 7 & 0 & 4 & 35 & 26 & 9 \\
\hline 1 & 5 & 243 & 243 & 6 & 3 & -6 & 174 & 170 & 5 & 0 & 6 & 114 & 112 & 4 \\
\hline 1 & 6 & 84 & 78 & 4 & 3 & -5 & 109 & 107 & 4 & 0 & 8 & 59 & 53 & 6 \\
\hline 1 & 7 & 138 & 136 & 5 & 3 & -4 & 171 & 170 & 5 & 0 & 12 & 215 & 213 & 7 \\
\hline 1 & 8 & 112 & 110 & 4 & 3 & -3 & 134 & 140 & 5 & 0 & 14 & 171 & 165 & 6 \\
\hline 1 & 9 & 187 & 193 & 6 & 3 & -2 & 83 & 74 & 5 & 0 & 16 & 49 & 51 & 9 \\
\hline 1 & 10 & 55 & 56 & 7 & 3 & 0 & 94 & 92 & 4 & 0 & 18 & 80 & 82 & 6 \\
\hline 1 & 11 & 92 & 86 & 4 & 3 & 1 & 155 & 157 & 5 & 1 & -17 & 105 & 100 & $E$ \\
\hline 1 & 14 & 52 & 52 & 8 & 3 & 2 & 64 & 65 & 6 & 1 & -16 & 122 & 124 & 4 \\
\hline 1 & 16 & 73 & 84 & 6 & 3 & 3 & 115 & 111 & 4 & 1 & -12 & 130 & 138 & 5 \\
\hline 1 & 19 & 70 & 83 & 7 & 3 & 4 & 114 & 114 & 4 & 1 & -11 & 71 & 78 & 6 \\
\hline 2 & -17 & 108 & 105 & 5 & 3 & 5 & 79 & 84 & 5 & 1 & -10 & 83 & 78 & 5 \\
\hline 2 & -15 & 185 & 190 & 6 & 3 & 6 & 88 & 83 & 5 & 1 & -9 & 55 & 60 & 7 \\
\hline 2 & -13 & 327 & 324 & 7 & 3 & 7 & 178 & 181 & 5 & 1 & -8 & 178 & 182 & 5 \\
\hline 2 & -12 & 85 & 85 & 5 & 3 & 8 & 138 & 137 & 5 & 1 & -7 & 92 & 95 & 4 \\
\hline 2 & -11 & 216 & 224 & 7 & 3 & 10 & 69 & 60 & 6 & 1 & -6 & 124 & 121 & 4 \\
\hline 2 & -10 & 388 & 379 & 8 & 3 & 12 & 124 & 129 & 4 & 1 & -4 & 101 & 104 & 4 \\
\hline 2 & -9 & 271 & 276 & 7 & 3 & 1.4 & 61 & 51 & 7 & 1 & -3 & 243 & 241 & 6 \\
\hline 2 & -8 & 101 & 102 & 4 & 3 & 16 & 122 & 131. & 5 & 1. & -2 & 291 & 288 & 6 \\
\hline 2 & -7 & 72 & 70 & 5 & 4 & -12 & 123 & 120 & 5 & 1 & -1 & 183 & 179 & 6 \\
\hline 2 & -6 & 197 & 189 & 6 & 4 & -10 & 63 & 61 & 7 & 1 & 0 & 50 & 44 & 6 \\
\hline 2 & -5 & 200 & 207 & 6 & 4 & -8 & 139 & 137 & 5 & 1 & 1. & 233 & 225 & 6 \\
\hline 2 & -3 & 129 & 127 & 4 & 4 & -6 & 208 & 202 & 7 & 1 & 2 & 171 & 170 & 6 \\
\hline 2 & -2 & 44 & 51 & 7 & 4 & -5 & 112 & 100 & 4 & 1 & 3 & 67 & 58 & 5 \\
\hline 2 & -1 & 283 & 291 & 6 & 4 & -4 & 161 & 153 & 6 & 1 & 4 & 46 & 44 & 7 \\
\hline 2 & 0 & 123 & 125 & 4 & 4 & -3 & 160 & 152 & 5 & 1 & 6 & 127 & 129 & 4 \\
\hline 2 & 1 & 88 & 82 & 4 & 4 & -2 & 75 & 72 & 6 & 1 & 7 & 250 & 244 & 6 \\
\hline 2 & 2 & 275 & 269 & 6 & 4 & -1 & 114 & 116 & 4 & 1 & 9 & 143 & 147 & 5 \\
\hline 2 & 3 & 87 & 87 & 4 & 4 & 0 & 46 & 49 & 8 & 1 & 10 & 58 & 55 & 6 \\
\hline 2 & 4 & 52 & 51 & 6 & 4 & 1 & 256 & 251 & 7 & 1 & 11 & 157 & 165 & 5 \\
\hline 2 & 5 & 58 & 59 & 6 & 4 & 3 & 73 & 69 & 6 & 1 & 12 & 39 & 31 & 10 \\
\hline 2 & 6 & 64 & 66 & 5 & 4 & 5 & 73 & 72 & 5 & 1 & 13 & 57 & 56 & 7 \\
\hline 2 & 7 & 49 & 62 & 7 & 4 & 6 & 73 & 73 & 6 & 1 & 14 & 120 & 122 & 4 \\
\hline 2 & 8 & 74 & 82 & 5 & 4 & 9 & 62 & 57 & 7 & 1 & 15 & 100 & 106 & 5 \\
\hline 2 & 9 & 244 & 242 & 6 & 4 & 11 & 86 & 80 & 5 & 2 & -15 & 103 & 100 & 5 \\
\hline 2 & 10 & 286 & 285 & 7 & 5 & -6 & 50 & 38 & 9 & 2 & -14 & 88 & 82 & 5 \\
\hline 2 & 11 & 61 & 61 & 7 & 5 & -3 & 95 & 91 & 5 & 2 & -13 & 285 & 292 & 7 \\
\hline 2 & 12 & 69 & 65 & 6 & 5 & 3 & 75 & 72 & 6 & 2 & -11 & 118 & 110 & 4 \\
\hline 2 & 13 & 144 & 144 & 5 & 5 & 4 & 54 & 53 & 8 & 2 & -10 & 162 & 160 & 5 \\
\hline 2 & 15 & 68 & 59 & 6 & & & & & & 2 & -8 & 181 & 186 & 5 \\
\hline 2 & 16 & 73 & 79 & 6 & $\wedge \wedge$ & ^^^ h & $=$ & $9 \wedge \wedge \wedge$ & $m n$ & 2 & -5 & 216 & 224 & 7 \\
\hline 2 & 17 & 72 & 60 & 7 & & & & & & 2 & -4 & 182 & 173 & 6 \\
\hline 2 & 18 & 100 & 111 & 5 & 0 & -1.6 & 130 & 130 & 5 & 2 & -2 & 76 & 70 & 5 \\
\hline
\end{tabular}


$10|\mathrm{~F}| \mathrm{O}$ VS $10|\mathrm{~F}| \mathrm{C}$

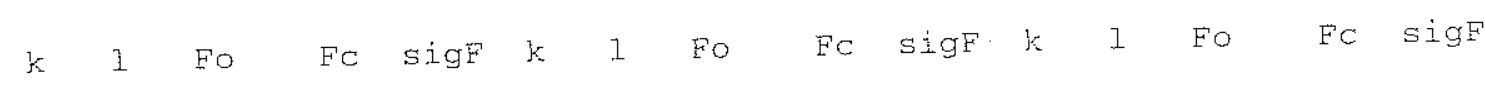

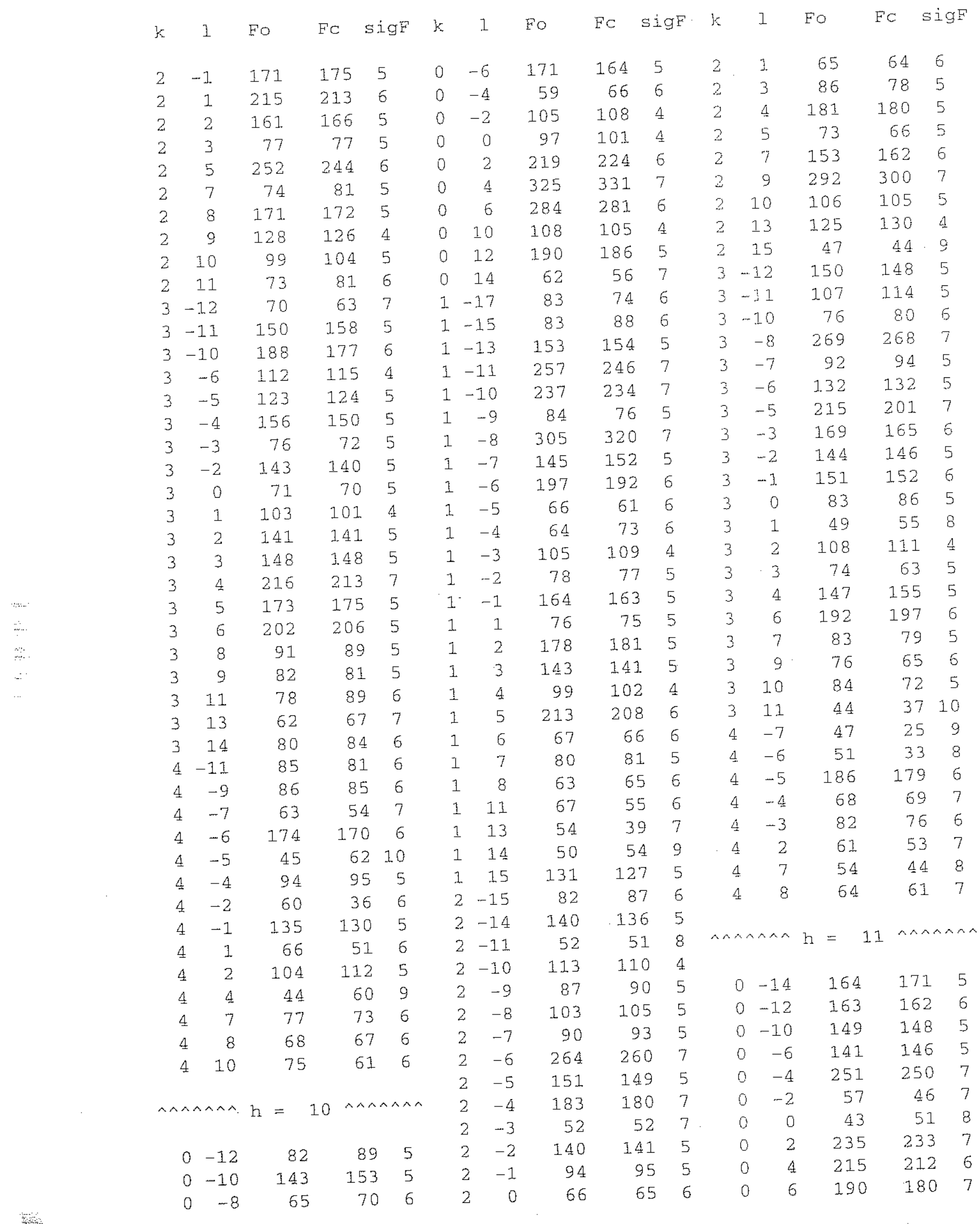


$10|F| O$ VS $10|F| C$

page 11

$k 1$ FO FC sigf $k 1$ FO FC sigF $k 1$ FO FC sigF

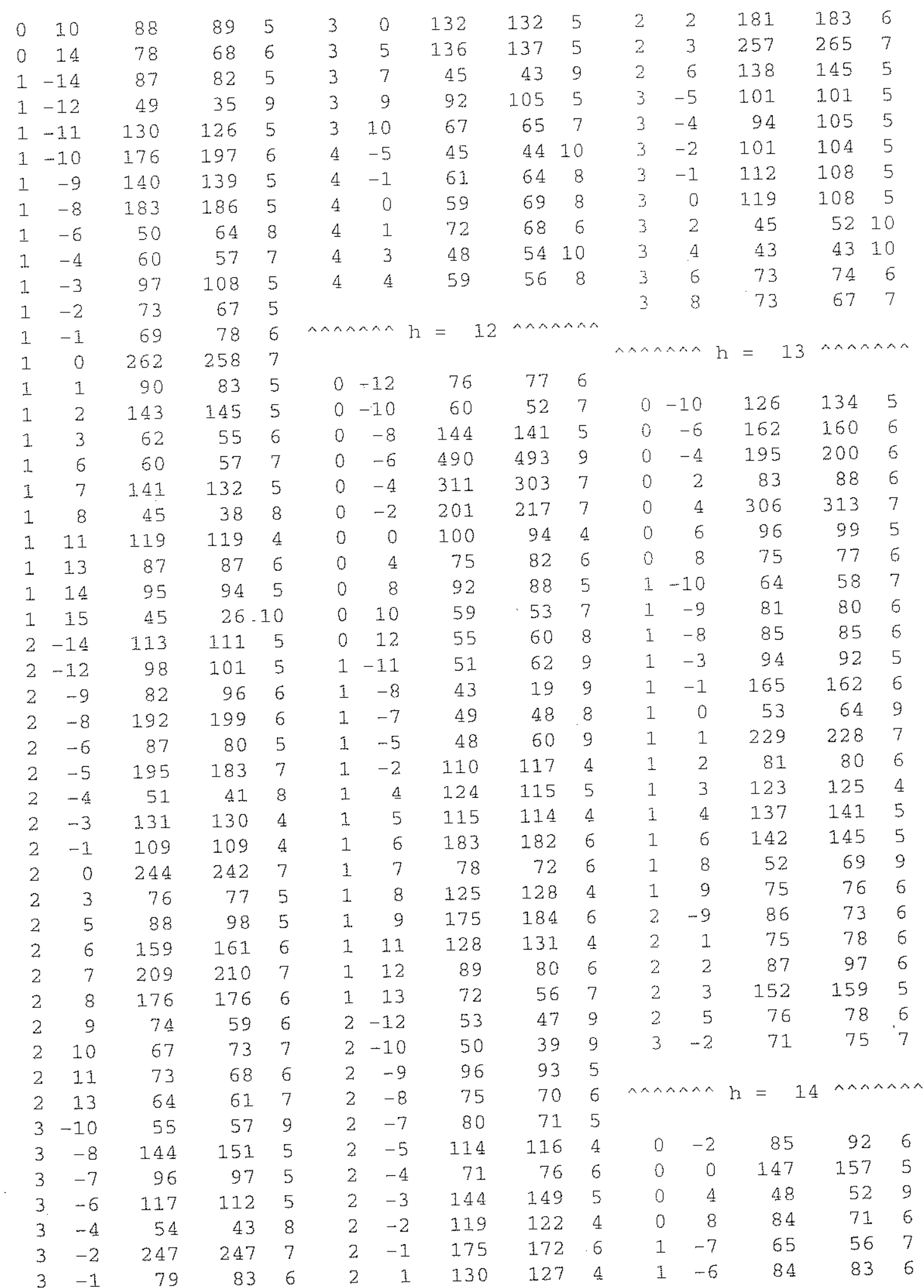




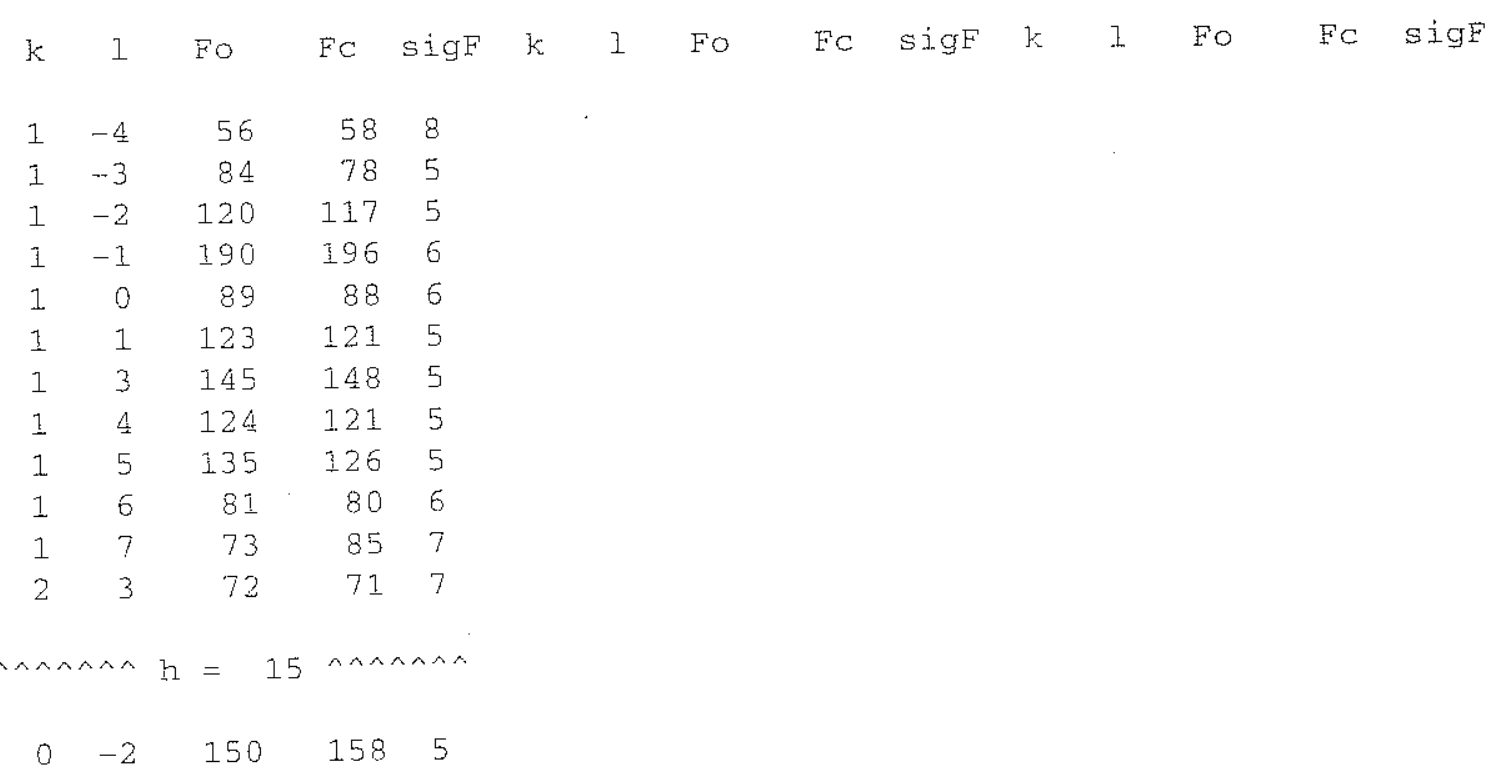

